\title{
An Application of the Hungarian Algorithm to Solve Traveling Salesman Problem
}

\author{
Janusz Czopik \\ Sugar Land, USA \\ Email: jczopik@comcast.net
}

How to cite this paper: Czopik, J. (2019) An Application of the Hungarian Algorithm to Solve Traveling Salesman Problem. American Journal of Computational Mathematics, 9, 6167.

https://doi.org/10.4236/ajcm.2019.92005

Received: April 23, 2019

Accepted: June 8, 2019

Published: June 11, 2019

Copyright (c) 2019 by author(s) and Scientific Research Publishing Inc.

This work is licensed under the Creative Commons Attribution International License (CC BY 4.0).

http://creativecommons.org/licenses/by/4.0/

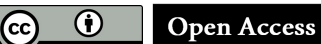

\section{Abstract}

In this paper we applicate the Hungarian algorithm for assignment problem to solve traveling salesman problem. Tree examples of application of algorithm are included.

\section{Keywords}

Traveling Salesman, Assignment Problem, Polynomial Time, Hungarian Algorithm

\section{Introduction}

The traveling salesman problem TSP according to Wikipedia [1] asks the following question: "Given a list of cities and the distances between each of pair of cities, what is the shortest possible route that visits each city exactly once and returns to the origin city?"

We will consider symmetric TSP that is the distance between two cities is the same in each opposite direction.

TSP is an NP-hard problem [2] in combinatorial optimization and can be used as a model to the variety of problems.

There exist many algorithms to solve this problem. Some of them gives approximate solutions using heuristic arguments, for example: genetic algorithm, paritcle swarm optimization algorithm, Christofides algorithm.

Another algorithms have a complexity of $O(n !)$. Our aim is to propose the exact algorithm which works in polynomial time [2]. Until now such algorithm not exists.

Salesman traveling problem is similar to the assignment problem AP for which polynomial time algorithm exists [3]. That algorithm is called "Hungarian algorithm" [4]. It was developed and published in 1955 by Harold Kuhn [5]. In 1957 J. Munkres [6] noted that it is a strongly polynomial algorithm. Later was modified to achieve $O\left(n^{3}\right)$ running time.

Now we formulate both problems mathematically. 


\section{Assignment Problem vs. Traveling Salesman Problem}

The assignment problem AP is to find a permutation matrix

$$
X=\left[x_{i j}\right]
$$

such that the sum $\sum_{i, j} x_{i j} r_{i j}$ is minimal, where $r_{i j}$ are the elements of the given matrix

$$
R=\left[r_{i j}\right] .
$$

The traveling salesman problem (TSP) is to find a permutation matrix

$$
X=\left[x_{i j}\right]
$$

such that a sum $\sum x_{i j} c_{i j}$ is minimal and

$$
C=\left[c_{i j}\right]
$$

is a distant matrix. Additionally, in matrix $X$ all elements on diagonal equal to zero and there exists a pair of elements

$$
(1, k)=1
$$

and

$$
(k, 1)=1 \text {. }
$$

We note that directed graph of $X$ contains a simple cycle of length $n$, that is a directed path that begins and ends at the same node, and not other node occurs more then once in the path.

To solve TSP we will use algorithm for AP.

We assume that $R$ is $n \times n$ matrix.

Algorithm for assgnment problem have following steps:

1). Fore each row of the matrix $R$, find the smallest element and substract it from every element in its row.

2 ). Do the same (as step1) for all columns.

3). Cover all zeros in the matrix using minimum number of horizontal and vertical lines.

4). If the minimum number of covering lines is $n$, an optimal assignment is possible and we are finished. Else if lines are lesser than $n$, we haven't found the optimal assignment, and must proceed to step 5 .

$5)$. Determine the smallest element not covered by any line. Substract this element from each uncovered row, and then add it to each covered column. Return to step 3.

\section{Algorithm for Traveling Salesman Problem}

1). On diagonal of matrix $C$ we set all elements equal to $\infty$.

2). We solve AP with modifying matrix $C$.

3 ). If the solution contains only single cycle TSP is solved. Unfortunately, optimal solution of AP may contains short cycles.

4). If appears more cycles then we starts to remove them.

The cycle appears if the solution contains pair of elements $(i, k)$ and $(l, i)$ different of 0 and $i \neq 1$. 
Then we put

$$
(i, k)=\infty
$$

or

$$
(l, i)=\infty .
$$

for each of cycle.

We solve AP with modifying matrix. If we get solution with only single cycle TSP is solved. If not we continue 4.

Remark. Sametimes is sufficient to remove only one cycle.

The full graph always contains single cycle. The cost of that cycle is fixed. It is upper limit of all cycles obtaining by AP Thus algorithm must be finite.

\section{Examples}

Example 1.

The distant matrix

$\begin{array}{ccccccc} & 1 & 2 & 3 & 4 & 5 & 6 \\ 1 & - & 12 & 29 & 22 & 13 & 24 \\ 2 & 12 & - & 19 & 3 & 25 & 6 \\ 3 & 29 & 19 & - & 21 & 23 & 28 \\ 4 & 22 & 3 & 21 & - & 4 & 5 \\ 5 & 13 & 25 & 23 & 4 & - & 16 \\ 6 & 24 & 6 & 28 & 5 & 16 & -\end{array}$

Solving(AP by (HA) we get solution of the form:

$(1,5)(2,3)(3,2)(4,6)(5,1)(6,4)$ with optimal value 74 .

We see that are three cycles.

Modifying distant matrix as

$\begin{array}{ccccccc} & 1 & 2 & 3 & 4 & 5 & 6 \\ 1 & \infty & 12 & 29 & 22 & 13 & 24 \\ 2 & 12 & \infty & \infty & 3 & 25 & 6 \\ 3 & 29 & 19 & \infty & 21 & 23 & 28 \\ 4 & 22 & 3 & 21 & \infty & 4 & 5 \\ 5 & 13 & 25 & 23 & 4 & \infty & 16 \\ 6 & 24 & 6 & 28 & 5 & 16 & \infty\end{array}$

and solving AP by (HA) we get optimal solution

$(1,3)(2,6)(3,2)(4,5)(5,1)(6,4)$.

In this example we remove only one cycle.

The calculation process is like.

This is original modifying cost matrix:

$\begin{array}{cccccc}1000 & 12 & 29 & 22 & 23 & 24 \\ 12 & 1000 & 100 & 3 & 25 & 6 \\ 29 & 19 & 1000 & 21 & 23 & 28 \\ 22 & 3 & 21 & 1000 & 4 & 5 \\ 13 & 25 & 23 & 4 & 1000 & 16 \\ 24 & 6 & 28 & 5 & 16 & 1000\end{array}$

Subtract row minima 
We subtract the row minimum from each row:

$\begin{array}{ccccccc}988 & 0 & 17 & 10 & 1 & 12 & (-12) \\ 9 & 997 & 97 & 0 & 22 & 3 & (-3) \\ 10 & 0 & 981 & 2 & 4 & 9 & (-19) \\ 19 & 0 & 18 & 997 & 1 & 2 & (-3) \\ 9 & 21 & 19 & 0 & 996 & 12 & (-4) \\ 19 & 1 & 23 & 0 & 11 & 995 & (-5)\end{array}$

substract column minima

We substract the column minimum from each column

$\begin{array}{cccccc}979 & 0 & 0 & 10 & 0 & 10 \\ 0 & 997 & 80 & 0 & 21 & 1 \\ 1 & 0 & 964 & 2 & 3 & 7 \\ 10 & 0 & 1 & 997 & 0 & 0 \\ 0 & 21 & 2 & 0 & 995 & 10 \\ 10 & 1 & 6 & 0 & 10 & 993 \\ (-9) & & (-17) & & (-1) & (-2)\end{array}$

Cover all zeros with a minimum number of llines

There are 5 lines requires to cover all zeros:

$\begin{array}{ccccccc}976 & 0 & 0 & 10 & 0 & 10 & x \\ 0 & 997 & 80 & 0 & 21 & 1 & \\ 1 & 0 & 964 & 2 & 3 & 7 & x \\ 10 & 0 & 1 & 997 & 0 & 0 & x \\ 0 & 21 & 2 & 0 & 995 & 10 & \\ 10 & 1 & 6 & 0 & 10 & 993 & \\ x & & & x & & & \end{array}$

Create additional zeros

The number of lines is smaller then 6 . The smallest uncovered number is 1 . We subtract this number from all uncovered elements and add it to all elements that are covered twice:

$\begin{array}{cccccc}980 & 0 & 0 & 11 & 0 & 10 \\ 0 & 996 & 79 & 0 & 20 & 0 \\ 2 & 0 & 964 & 3 & 3 & 7 \\ 11 & 0 & 1 & 998 & 0 & 0 \\ 0 & 20 & 1 & 0 & 994 & 9 \\ 10 & 0 & 5 & 0 & 9 & 992\end{array}$

Cover all zeros with a minimum number of lines There are 6 lines requires to cover all zeros:

$\begin{array}{ccccccc}980 & 0 & 0 & 11 & 0 & 10 & x \\ 0 & 996 & 79 & 0 & 20 & 0 & x \\ 2 & 0 & 964 & 3 & 3 & 7 & x \\ 11 & 0 & 1 & 998 & 0 & 0 & x \\ 0 & 20 & 1 & 0 & 994 & 9 & x \\ 10 & 0 & 5 & 0 & 9 & 992 & x\end{array}$

The optimal assignment

Because there are 6 lines required, the zeros cover an optimal assignment:

$\begin{array}{cccccc}960 & 0 & \mathbf{0} & 11 & 0 & 10 \\ 0 & 996 & 79 & 0 & 20 & \mathbf{0} \\ 2 & \mathbf{0} & 964 & 3 & 3 & 7 \\ 11 & 0 & 1 & 998 & \mathbf{0} & 0 \\ \mathbf{0} & 20 & 1 & 0 & 994 & 9 \\ 10 & 0 & 5 & \mathbf{0} & 9 & 992\end{array}$


This corresponds to the following optimal assignment in the original cost matrix:

$\begin{array}{cccccc}1000 & 12 & \mathbf{2 9} & 22 & 13 & 24 \\ 12 & 1000 & 1000 & 3 & 25 & \mathbf{6} \\ 29 & \mathbf{1 9} & 1000 & 21 & 23 & 28 \\ 22 & 3 & 21 & 1000 & \mathbf{4} & 5 \\ \mathbf{1 3} & 25 & 23 & 4 & 1000 & 16 \\ 24 & 6 & 28 & \mathbf{5} & 16 & 1000\end{array}$

The optimal value equals 76 .

Example 2. From "Traveling Salesman Problem" - University of Washington.

We have distant matrix of the form:

$\begin{array}{lllllll} & 1 & 2 & 3 & 4 & 5 & 6 \\ 1 & & 1 & 5 & 4 & & \\ 2 & 1 & & 2 & 2 & & 2 \\ 3 & & 2 & & 1 & 4 & 1 \\ 4 & 4 & 2 & 1 & & 2 & 2 \\ 5 & & & 4 & 2 & & 3 \\ 6 & & 2 & 1 & 2 & 3 & \end{array}$

Some cities are not connected.

This is not classical TSP. Nevertheless we can solve it using our algorithm. First we rewrite the distant matrix as follows:

$\begin{array}{ccccccc} & 1 & 2 & 3 & 4 & 5 & 6 \\ 1 & \infty & 1 & 5 & 4 & \infty & \infty \\ 2 & \infty & 2 & 2 & \infty & 2 & \\ 3 & \infty & 2 & \infty & 1 & & 1 \\ 4 & 4 & 2 & 1 & \infty & 2 & 2 \\ 5 & \infty & \infty & 4 & 2 & \infty & 3 \\ 6 & \infty & 2 & 1 & 2 & 3 & \infty\end{array}$

Then we solve AP with modifying matrix by (HA).

The solution is of the form:

$(1,2)(2,1)(3,6)(4,5)(5,4)(6,3)$ with optimal value 13.

We see 3 small cycles in solution.

Processing as in Ex. 1 we obtain the matrix:

$\begin{array}{ccccccc} & 1 & 2 & 3 & 4 & 5 & 6 \\ 1 & \infty & 1 & 5 & 4 & \infty & \infty \\ 2 & \infty & \infty & 2 & 2 & \infty & 2 \\ 3 & \infty & 2 & \infty & 1 & 4 & 1 \\ 4 & 4 & 2 & 1 & \infty & 2 & 2 \\ 5 & \infty & \infty & 4 & 2 & \infty & 3 \\ 6 & \infty & 2 & 1 & 2 & 3 & \infty\end{array}$

Using (HA) we get the optimal solution

$(1,2)(2,3)(3,6)(4,1)(5,4)(6,5)$

The optimal value equals 13 . 
Example 3. From []

$\begin{array}{ccccccccc} & 1 & 2 & 3 & 4 & 5 & 6 & 7 & 8 \\ 1 & \infty & 2 & 11 & 10 & 8 & 7 & 6 & 5 \\ 2 & 6 & \infty & 1 & 8 & 8 & 4 & 6 & 7 \\ 3 & 5 & 12 & \infty & 11 & 8 & 12 & 3 & 11 \\ 4 & 11 & 9 & 10 & \infty & 1 & 9 & 8 & 10 \\ 5 & 11 & 11 & 9 & 4 & \infty & 2 & 10 & 9 \\ 6 & 12 & 8 & 5 & 2 & 11 & \infty & 11 & 9 \\ 7 & 10 & 11 & 12 & 10 & 9 & 12 & \infty & 3 \\ 8 & 7 & 10 & 10 & 10 & 6 & 3 & 1 & \infty\end{array}$

First we solve AP for this example.

Solution is of the form:

$(1,2)(2,3)(3,1)(4,5)(5,6)(6,4)(7,8)(8,7)$

We see tree cycles. Modifying cost matrix as:

$\begin{array}{cccccccc}100 & 100 & 11 & 10 & 8 & 7 & 6 & 5 \\ 5 & 100 & 1 & 8 & 8 & 4 & 6 & 7 \\ 5 & 12 & 100 & 11 & 8 & 12 & 3 & 11 \\ 11 & 9 & 10 & 100 & 100 & 9 & 8 & 10 \\ 11 & 11 & 9 & 4 & 100 & 2 & 10 & 9 \\ 12 & 8 & 5 & 2 & 11 & 100 & 11 & 9 \\ 10 & 11 & 12 & 10 & 9 & 12 & 100 & 100 \\ 7 & 10 & 10 & 10 & 6 & 3 & 1 & 100\end{array}$

and solving (HA) we get optimal TSP solution:

$(1,8)(2,3)(3,1)(4,2)(5,6)(6,4)(7,5)(8,7)$

The optimal value equals 34 .

In this example we removed all three cycles.

\section{Conclusion}

The aim of this article is to present an idea of strict, polynomial time algorithm for traveling salesman problem. The examples are shown that proposal algorithm works. The running time of this algorithm is as the algorithm for assignment problem. This algorithm is not supposed to be compared with probabilistic ones. We can find the comparison of the algorithms for solving traveling salesman problems in [7]. This algorithm as a new one, needs same development in the future.

\section{Conflicts of Interest}

The author declares no conflict of interest regarding the publication of this paper.

\section{References}

[1] https://en.wikipedia.org/wiki/Travelling-salesman-problem

[2] https://en.wikipedia.org/wiki/Hungarian-algorithm

[3] https://en.wikipedia.org./wiki/Assignment-problem

[4] http://www.hungarianalgorithm.com/

[5] Kuhn, H. (1955) The Hungarian Method for the Assignment Problem. Naval Research Logistics Quarterly, 2, 83-97. https://doi.org/10.1002/nav.3800020109 
[6] Munkres, J. (1957) Algorithms for the Assignment and Transportation Problems. Journal of the Society for Industrial and Applied Mathematics, 5, 32-38. https://doi.org/10.1137/0105003

[7] Abdulkarim, H.A. and Alshammari, I.F. (2015) Comparison of Algorithms for Solving Traveling Salesman Problem. International Journal of Engineering and Advanced Technology, 4, 76-79. 